\title{
A Bi-level Approach to Distribution Network and Renewable Energy Expansion Planning considering Demand Response
}

\author{
Miguel Asensio, Gregorio Muñoz-Delgado, Student Member, IEEE, and Javier Contreras, Fellow, \\ IEEE
}

\begin{abstract}
This paper presents a bi-level model for distribution network and renewable energy expansion planning under a Demand Response (DR) framework. The role of DR has recently attracted an increasing interest in power systems. However, previous models have not been completely adapted in order to treat DR on an equal footing. The target of the distribution network and generation planner, modeled through the upperlevel problem, is to minimize generation and network investment cost while meeting the demand. This upper-level problem is constrained by the lower-level problem, stressing the importance of integrating DR to time-varying prices into those investment models. The objective function considered for the lower level is the minimization of overall payment faced by the consumers. Using the Karush Kuhn Tucker (KKT) complementarity constraints, the proposed bi-level model is recast as a mixedinteger linear programming problem, which is solvable using efficient off-the-shelf branch-and-cut solvers. Detailed results from an insular case study (La Graciosa, Canary Islands, Spain) are presented.
\end{abstract}

Index Terms-DR, Renewable Energy Sources (RES) expansion planning, distribution network expansion planning, bilevel programming, KKT complementarity constraints.

\section{NOMENCLATURE}

$\begin{array}{ll}\text { Indices and Sets } \\ b & \text { Index for substation load levels } \\ B & \text { Set of substation load levels for the calculation } \\ & \text { of the substation price } \\ i, j & \text { Indices for nodes } \\ l & \text { Index for feeder types } \\ L & \text { Set of feeder types, } L=\{E F F, E R F, N R F, N A F\} \\ & \text { where } E F F, E R F, N R F \text { and } N A F \text { denote existing } \\ & \text { fixed feeders, existing replaceable feeders, new } \\ & \text { replacement feeders, and newly added feeders, } \\ & \text { respectively } \\ l l, l b & \text { Indices for load levels } \\ L L, L B & \text { Sets of load levels } \\ p & \text { Index for generator types } \\ P & \text { Set of generator types, } P=\{W, \Theta\} \text { where } \\ & W \text { and } \Theta \text { denote wind and photovoltaic } \\ t & \text { generators, respectively } \\ & \text { Index for time stages }\end{array}$

This work was supported in part by the Ministry of Economy and Competitiveness of Spain, under Project ENE2015-63879-R (MINECO/FEDER, EU), and the Junta de Comunidades de Castilla-La Mancha, under Project POII-2014-012-P and Grant PRE2014/8064.

The authors are with the Escuela Técnica Superior de Ingenieros Industriales, Universidad de Castilla-La Mancha, 13071 Ciudad Real, Spain (e-mail: miguel.asensio@gmail.com; gregorio.munoz.delgado@gmail.com; Javier.Contreras@uclm.es).
$T$
tr
Set of time stages
Index for transformer types
$T R \quad$ Set of transformer types, $T R=\{E T, N T\}$ where $E T$ and $N T$ denote existing transformers and newly added transformers, respectively
$w \quad$ Index for scenarios
$Y^{l} \quad$ Set of branches with feeders of type $l$
$\Omega_{i}^{l}, \Omega^{L N}, \quad$ Sets of nodes connected to node $i$ by a feeder $l$,
$\Omega^{N}, \Omega^{p}, \quad$ load nodes, system nodes, candidate nodes for
$\Omega^{s s} \quad$ DG, and substation nodes

Parameters

$C_{b} \quad$ Costs for substation load level $b$ [\$/MWh]

$C_{i, j}^{I, l} \quad$ Investment cost of feeder $l$ in branch $i-j[\$ / \mathrm{km}]$

$C_{i, j}^{M, l} \quad$ Maintenance cost of feeder $l$ in branch $i-j[\$]$

$C_{i}^{I, N T} \quad$ Investment cost of adding a new transformer at substation node $i$ [\$]

$C_{i}^{I, p} \quad$ Investment cost of generator $p$ at node $i$ [\$/MW]

$C_{i}^{M, p} \quad$ Maintenance cost of generator $p$ at node $i[\$]$

$C_{i}^{M, t r} \quad$ Maintenance cost of transformer $t r$ at substation node $i[\$]$

$C^{E, p} \quad$ Generation costs of generator type $p$ [\$/MWh]

$\overline{C_{t, l l, w}^{S S}} \quad$ Average substation cost at stage $t$ for load level

$l l$ in scenario $w$ considering adjacent blocks [\$/MWh]

$\widetilde{D}_{i, t} \quad$ Fictitious nodal demand at substation node $i$ at stage $t$

$D_{i, t, l l, w} \quad$ Expected demand at node $i$ at stage $t$ for load

level $l l$ in scenario $w$ [MVA]

$\bar{F}_{i, j}^{l} \quad$ Maximum capacity of feeder $l$ in branch $i-j$

[MVA]

$\bar{G}_{i}^{t r} \quad$ Upper limit for energy supplied by transformer

$\quad t r$ at substation node $i$ [MVA]

$\bar{G}^{p} \quad$ Maximum capacity of generator type $p$ [MVA]

I Annual investment rate

$I B_{t} \quad$ Investment budget for stage $t$ [\$]

$\ell_{i, j} \quad$ Feeder length [km]

$L_{b}{ }_{\text {min }} \quad$ Lower limit of substation load level $b$ [MVA]

$L_{b}{ }^{\max } \quad$ Upper limit of substation load level $b$ [MVA]

$n_{D G}, n_{T} \quad$ Number of candidate nodes for distributed generation, number of time stages

pf System power factor

$R R^{l}, R R^{N T}$ Capital recovery rates for investment in feeders, $R R^{p}, R R^{S S}$ new transformers, generators and substations

$Z_{i, j}^{l} \quad$ Unitary impedance magnitude of feeders [ $[\Omega]$ 
$\begin{array}{ll}\Delta_{l l}, \Delta_{l b} & \text { Duration of each load level } l l[\mathrm{~h}] \\ \varepsilon & \text { Penetration limit for distributed generation [\%] } \\ \xi_{l l l l}, \xi_{l b, l l} & \text { Own and cross price elasticities } \\ \pi_{l l, w} & \text { Probability of scenario } w \text { of load level } l l \\ v^{l}, v^{N T}, & \text { Lifetimes of feeders, new transformers, } \\ v^{p}, v^{s s} & \begin{array}{l}\text { generators and substation assets other than } \\ \text { transformers [yr] }\end{array}\end{array}$

Variables

$d_{i, t, l l, w} \quad$ Demand at node $i$ at stage $t$ for load level $l l$ in scenario $w$ when considering DR [MVA]

$d_{i, t, l l, w}^{U} \quad$ Unserved power at node $i$ at stage $t$ for load level $l l$ at scenario $w$ [MVA]

$d r_{i, t, l l, w} \quad$ DR at node $i$ stage $t$ for load level $l l$ in scenario $w$ [MVA]

$d r_{i, t, l l, w}^{+} \quad$ Upper and lower DR limits at node $i$ at stage $t$

$d r_{i, t, l l, w}^{-} \quad$ for load level $l l$ in scenario $w$ [MVA]

$f_{i, j, t, l l, w}^{l} \quad$ Current flow through feeder $l$ installed in branch $i-j$ at stage $t$, load level $l l$ in scenario $w$, measured at node $i$, which is greater than 0 if node $i$ is the supplier and 0 , otherwise [MVA]

$\tilde{f}_{i, j, t}^{l} \quad$ Fictitious current flow through feeder $l$ in branch $i-j$ at stage $t$, measured at node $i$, which is greater than 0 if node $i$ is the supplier and 0 , otherwise

$g_{i, t, l l, w}^{p} \quad$ Energy supplied by generator $p$ at node $i$ at stage $t$ for load level $l l$ in scenario $w$ [MVA]

$g_{i, t, l l, w}^{t r} \quad$ Energy supplied by transformer $t r$ at substation node $i$ at stage $t$ for load level $l l$ in scenario $w$ [MVA]

$p_{t, l l, w} \quad$ Demand payment at stage $t$ for load level $l l$ in scenario $w$ [MVA]

$v_{i, t, l l, w} \quad$ Nodal voltage at node $i$ at stage $t$, load level $l l$ in scenario $w$

$x_{i, j, t}^{l} \quad$ Binary variable for the installation of feeder $l$ installed in branch $i-j$ at stage $t$

$x_{i, t}^{N T} \quad$ Binary variable for the installation of new transformers at substation node $i$ at stage $t$

$x_{i, t}^{p} \quad$ Binary variable for the installation of generator type $p$ at nodes $i$ at stage $t$

$x_{i, t}^{S S} \quad$ Binary variable for the reinforcement of existing substations or construction of new ones at substation node $i$ at stage $t$

$y_{i, j, t}^{l}, y_{i, t}^{p}$, Binary utilization variables for feeders, $y_{i, t}^{t r} \quad$ generators and transformers

$z_{t, l l, w}^{d r} \quad$ Binary variable associated with DR at stage $t$ for load level $l l$ in scenario $w$

$z_{t, b, l l, w} \quad$ Binary variable associated with substation prices at substation load level $b$ stage $t$ for load level $l l$ in scenario $w$

$\lambda^{1}{ }_{i, t, l l, w}$,

$\lambda^{2}{ }_{i, t, w}$

$\mu_{i, t, l l, w}^{1}$,

$\mu^{2}{ }_{i, t, l l, w}$

$\theta_{t, b, l l, w}$

Lagrange multipliers

Variable associated with substation demand at stage $t$ for substation load level $b$ and load level $l l$ in scenario $w$

$\rho_{t, b, l l, w} \quad$ Variable associated with substation demand at stage $t$ for substation load level $b$ and load level

\section{$l l$ in scenario $w$}

\section{INTRODUCTION AND CONTRIBUTIONS}

$\mathrm{D}^{\mathrm{E}}$ EMAND response and dynamic pricing are often considered as means of mitigating the uncertainties and intermittencies of renewable generation and improving the system's efficiency with respect to economic and environmental metrics. The idea is to allow the consumers to adjust their consumption in response to a signal that reflects the wholesale market conditions, possibly the real-time prices. Characterized by passing on the real-time wholesale electricity prices to the end consumers, real-time pricing creates a closedloop feedback system between the physical layer and the market layer of the system. In recent years, a large number of research works have been carried out on generation and distribution expansion planning. These problems have been investigated with different views, methods, constraints and objectives. However, these models have not been completely adapted in order to treat price-dependent resources, such as $\mathrm{DR}$, on an equal footing. We present a long-term distribution network and renewable energy expansion planning model, that considers short-term real time pricing demand response, i.e., as the electricity price increases, consumers reduce their demand and vice versa. Electricity demand can often no longer be assumed to be a fixed quantity, because demand response programs allow consumers to respond to electricity prices and adapt their electricity consumption accordingly. In economic theory, this is equivalent to a downward sloping demand function to represent the bidding behavior of electricity consumers. Several research works have been devoted to outline the advantages of integrating renewable technologies in the distribution network. The proposed approach bases on the findings of [1], where a model to obtain the optimal allocation and sizing of distributed generation units and distribution network assets while minimizing network investments is presented. The model described in [2] proposes an integrated methodology for distribution network expansion planning incorporating distributed generation considering reliability. The methods in [1] and [2] present a comprehensive literature review on previous works performed, including [3][7]. The distribution network expansion problem is analyzed through a heuristic algorithm in [3]. Authors in [4] present a probabilistic approach to distribution system planning. Authors in [5] present a mixed-integer quadratically-constrained model to solve the distribution system expansion planning problem. The formulation presented in [6] presents the mathematical model of considering DG on the expansion planning of a subtransmission system. A practical method is proposed in [7], combining Successive Linear Programming with Gauss-Seidel iteration to co-optimize $\mathrm{AC}$ and $\mathrm{DC}$ transmission and generation capacities in a linearized DC network. The effects of integrating short-term DR into long-term generation investment planning are investigated in [8]. Authors in [9] present an integrated methodology for planning distribution networks in which the operation of distributed generators (DGs) and cross-connections (CCs) is optimally planned. The effects of integrating DR into long-term generation and transmission network investment planning are investigated in [10] through a probabilistic multi-objective function. Authors in [11] present an analytical framework to incorporate DR in 
long-term resource planning. A symmetric treatment of load and generation creates the strongest possible incentive for final consumers to actively participate in the wholesale electricity market. The proposed approach translates this argument to medium- and long-term distribution and generation expansion planning.

Bi-level programming is considered an appropriate framework to model optimization problems in which one or several constraints are optimization problems themselves [12], [13]. Therefore the objective function of the upper-level problem is optimized considering that the lower-level problem optimizes its own objective function. Bi-level programming is proposed as a suitable solution methodology for the generation and distribution expansion planning problem, considering the expansion planning in the upper level and decisions associated to the flexibility of the demand in the lower level. Unlike the formulation proposed in [14], in which the operation of electric power systems where the energy purchase from DG and substations is minimized, the proposed approach minimizes the payment of the consumers in a long-term planning problem. Unlike previous works [1]-[10], the proposed programming formulation explicitly takes into consideration the minimization of consumers' payment (lower-level problem), incorporating DR into the long-term expansion planning. An extensive study about elasticities is performed in [15]. Based on [15] and [16], sufficiently accurate estimations for consumers' behavior have been included, quantifying the relationship between demand and market prices. Several methods have been proposed in the literature targeting the aforementioned problems. However, in our opinion, none of the existing works sufficiently stresses the relevance of DR in the expansion planning modeling. The proposed approach is based on the work performed in [17], enhancing the generation and distribution network expansion planning incorporating the bi-level formulation in order to adequately account for consumer's flexibility. Table I summarizes the main differences between this paper and the state of the art on multi-stage co-optimized expansion planning [1]-[10]. According to the presented state of the art, the following contributions can be listed:

- A novel multi-stage model is proposed to accurately include DR in the joint distribution and generation expansion planning problem through a bi-level formulation. Timing, location, and sizing of DG units and distribution network assets are modeled. The model proposes a bi-level formulation to incorporate price-dependent resources.

- The present model stresses the importance of integrating DR to time-varying prices (real-time prices) into those investment models. Own-price and cross-price elasticities are included in order to incorporate consumers' willingness to adjust the demand profile in response to price changes. Own price elasticity is defined as the change in the load of a certain level with respect to a change in price in the same load level $\left(\xi_{l l, l l}\right)$. Analogously, cross-price elasticity represents the change in the load of a certain level with respect to a change in price in other load levels $\left(\xi_{l b, l l}\right)$.

- Threats experienced by isolated systems as a consequence of increasing DG penetration are even higher than those experienced by interconnected systems since they cannot depend on the smoothing effect of a large balancing area. The novelty of the proposed approach is to stress the relevance of DR in isolated systems.

- A scenario-based stochastic programming framework is proposed to model the correlated uncertainty characterizing demand and renewable-based power generation. The associated deterministic equivalent is formulated as a mixed-integer linear program suitable for commercially available software.

The rest of this paper is organized as follows. Section II describes the proposed formulation, including substation price calculation and demand payment. In Section III, the test system of La Graciosa and the corresponding results are presented as a validation of the proposed model. Finally, some relevant conclusions are drawn in Section IV.

\section{PROBLEM FormuLATION}

The modeling of uncertainty in a distribution system for each single time block is based on the load, wind speed and solar irradiation duration curves. Hourly historical data are expressed as per-unit factors. Then, demand factor data are arranged from higher to lower values keeping the correlation between the different hourly data of wind speed and solar irradiation. The load duration curve is approximated using time blocks. In order to accurately include peak demand in the model, the length of the time blocks varies along the load duration curve. For each time block, wind speed and solar irradiation levels are arranged from higher to lower values (Fig. 2). In order to reduce the loss of information on the sequence of individuals when composing the load duration curve, demand, wind speed, and solar irradiation curves have been split into 48 different blocks based on quarter (4), weekend/working-day (2), day/night (2) and time blocks (3) criteria. For each time block, all combinations of demand, wind speed and solar irradiation levels are considered. Each combination is assigned a probability within the time block, $\pi_{l l, w}$, equal to the probability of the demand factor times the probability of the wind speed factor and the solar irradiation factor. For the present paper, 3 factor levels for demand, 3 factor levels for wind power and 3 factor levels for solar irradiation have been considered within each time block leading to 1,296 operating conditions per stage.

This procedure is based on the work proposed in [18]. The approach described in [18], and extended in [1] and [19], has revealed an effective performance to model the relation between demand, wind speed and solar irradiation. The methodology can be described in five steps:

Step 1) Firstly, historical values are separated into four groups of seasonal data, where working day/weekend and day/night separation has been performed in order to adequately represent the considered scenarios, resulting in 16 different sets of data that will be separately arranged following the next steps. Demand, wind speed, and solar irradiation are not statistically independent magnitudes. For each of the 16 data sets in a year the statistical interrelations are specifically accounted for while maintaining the correlation among them. Each of the considered data sets is divided by annual peak demand, maximum annual wind speed, and maximum annual solar irradiation, respectively, to obtain the factorized data. 
TABLE I

COMPARISOn OF Multi-Stage CO-OPTIMIZEd EXPANSION PlanNING Models

\begin{tabular}{cccccc}
\hline Approach & Topological changes & Renewable generation & Correlation & Demand Response & Bilevel Formulation \\
\hline$[1]$ & $\checkmark$ & $\checkmark$ & $\checkmark$ & $\times$ & $\times$ \\
{$[2],[6]$} & $\checkmark$ & $\checkmark$ & $\times$ & $\times$ & $\times$ \\
{$[3],[5]$} & $\checkmark$ & $\times$ & $\checkmark$ & $\times$ & $\times$ \\
{$[4],[9]$} & $\times$ & $\checkmark$ & $\checkmark$ & $\checkmark$ & $\times$ \\
{$[7],[8]$} & $\times$ & $\checkmark$ & $\checkmark$ & $\times$ & \\
{$[10]$} & $\checkmark$ & $\checkmark$ & $\checkmark$ & $\checkmark$ & \\
Proposed approach & $\checkmark$ & $\checkmark$ & & $\times$ \\
\hline
\end{tabular}

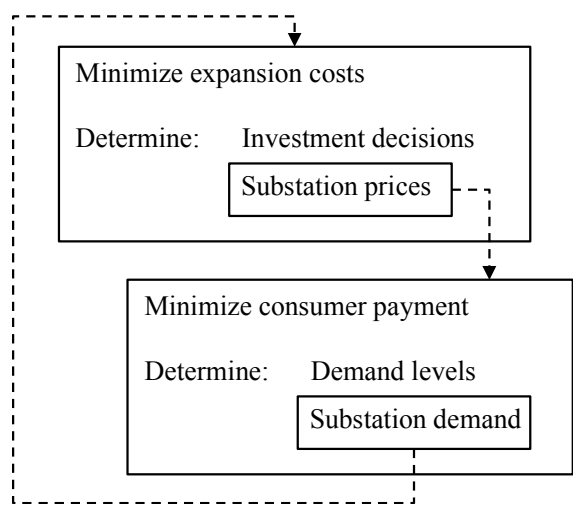

Fig. 1. Structure of the bi-level problem considered.

Step 2) For every data set, the demand curve is built sorting data in descending order keeping the hourly correlation between demand, wind speed and solar irradiation. Triplets of hourly factors for demand, wind speed, and solar irradiation are sorted by demand factor in descending order thereby creating a load duration curve for each data set, thus losing the chronology of the considered data.

Step 3) Time blocks are set to model the impact of the demand values.

Step 4) Wind speed and solar irradiation are ordered from highest to lowest in each time block. Then, for each time block and type of data (demand, wind speed, and solar irradiation), a cumulative distribution function is calculated. The proposed formulation provides a sufficiently accurate representation of the demand, wind speed and solar irradiation data within the considered blocks.

Step 5) The distribution function obtained is divided into segments. As depicted in Fig. 2, a total of three segments are considered. Each segment has an associated probability obtained from the corresponding cumulative distribution function. The system scenarios are formed by combining the levels obtained when modeling data uncertainty within each time block. Wind speed and solar irradiation values are then converted to power output values for wind and PV technologies.

Uncertainty sources, namely demand, wind speed, and solar irradiation are characterized by a set of scenarios that are generated on the basis of a previously reported approach relying on historical data [18]. Authors in [1] and [19] have extended the work described in [18] solving the multi-stage expansion planning problem. The approach proposed in the present formulation enhances [1] and [19] in order to adequately integrate time-dependent resources in the expansion planning. In this sense, the annual pattern is repeated for every block considered in the formulation. Therefore, the probability and level of each scenario in every stage is considered constant. According to [20], although the variability of the wind speed is site specific, it tends to be similar and can reasonably be assumed to be about $6 \%$. Similar results are provided in [21] accounting for the inter-annual variability of solar irradiation. Based on these claims, the assumption of constant probability for the considered scenarios among the different stages is deemed sufficient in order to construct the scenario tree for all stages. As formulated, the model is very flexible and can be easily extended to interconnected systems.
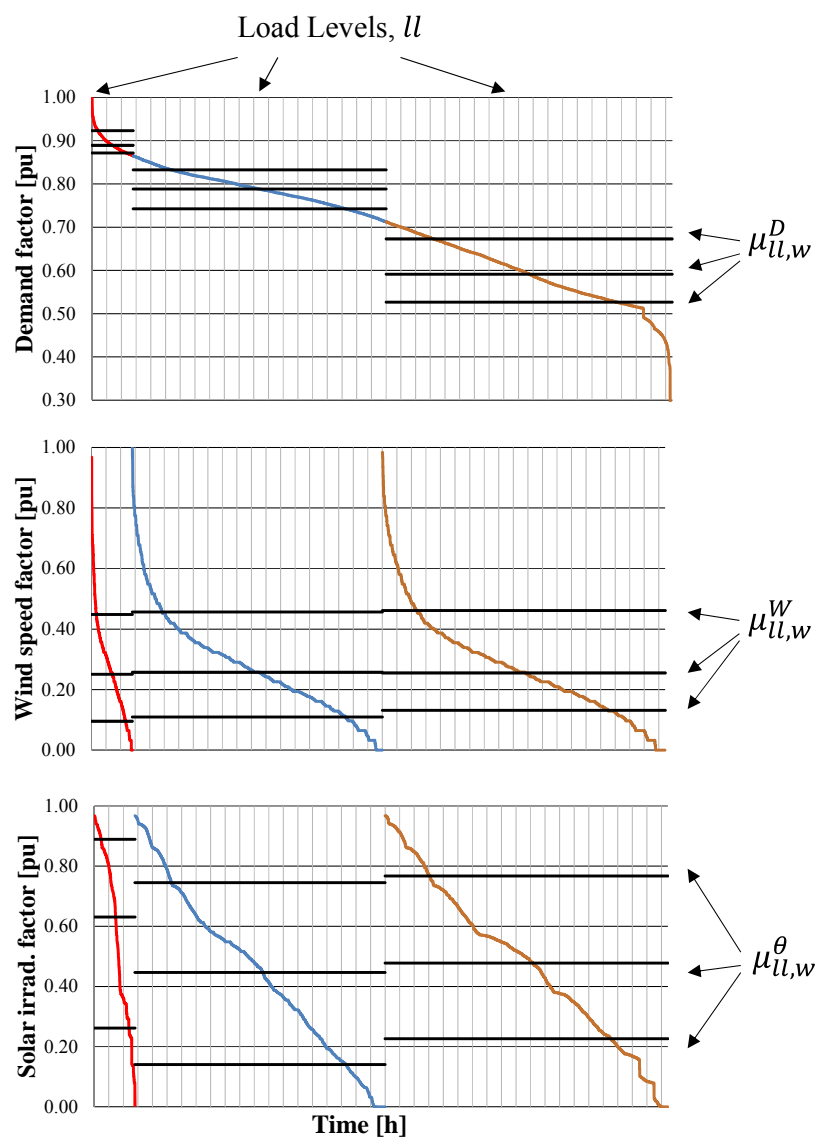

Fig. 2. Load, wind speed and solar irradiation duration curves for the summer/working-day/day scenario.

For each load level $l l$, each scenario $w$ comprises an average demand factor $\mu_{l l, w}^{D}$, an average level of wind speed $\mu_{l l, w}^{W}$, and an average level of solar irradiation $\mu_{l l, w}^{\theta}$. Nodal demands in 
each scenario are equal to the product of the forecasted values and the demand factor $\mu_{l l, w}^{D}$ throughout the planning horizon giving rise to $D_{i, t, l l, w}$. Moreover, for each scenario $w, \mu_{l l, w}^{W}$ and $\mu_{l l, w}^{\theta}$ are converted to maximum levels of wind and photovoltaic power generation, $\hat{G}_{i, t, l l, w}^{W}$ and $\hat{G}_{i, t, l l, w}^{\theta}$, respectively. For the sake of simplicity, we consider that $\mu_{l l, w}^{W}$ and $\mu_{l l, w}^{\theta}$ are identical for all candidate nodes and all considered time stages.

The distribution network and renewable generation expansion planning can be formulated in a compact way as:

$\min _{x, y} F(x, y)$

subject to:

$x \in X$

$G^{e}(x, y)=0$

$G^{i}(x, y) \geq 0$

where vector $x$ represents the upper-level variables, whereas vector $y$ characterizes the reaction of final consumers to timevarying prices. The objective function (A1) comprises the total cost, including investment, maintenance, energy purchase from substations and DG production and unserved power. Expressions (A2), exclusively involving investment variables, include network, generation, and investment constraints. Constraints (A3) denote the equalities associated to the model including Kirchhoff's law constraints. Constraints (A4) model all inequalities involving variables such as power flow limits and generation limits.

The minimization of the consumer's payment is an optimization problem by itself (A5)-(A7) which can be formulated for the optimal vector of investment variables $x^{*}$ :

$\min _{y} f\left(x^{*}, y\right)$

subject to:

$G^{E}\left(x^{*}, y\right)=0:(\lambda)$

$G^{I}\left(x^{*}, y\right) \geq 0:(\mu)$

where the Lagrange multipliers associated with constraints (A6) and (A7) are in parentheses. Thus, the distribution network and renewable generation expansion planning considering DR formulated in (A1)-(A4) can be recast as the following bi-level programming problem:

$\min _{x} F\left(x, y^{*}\right)$

subject to:

$x \in X$

$H\left(x, y^{*}\right) \geq 0$

where $y^{*}$ is obtained from

$\min _{y} f\left(x^{*}, y\right)$

subject to:

$G^{e}\left(x^{*}, y\right)=0:(\lambda)$

$G^{i}\left(x^{*}, y\right) \geq 0:(\mu)$

The upper level controls the upper-level decision variables $x$. Lower-level decision variables $y$ characterize the reaction of final consumers to time-varying prices. The objective of the upper level is the minimization of the net present value of the total cost (including investment, maintenance, energy purchase from substations and DG production and unserved power), evaluated at the optimal values of the lower-level variables. Substation prices can be accurately approximated by a set of price levels (Fig. 3). The proposed formulation assumes incremental substation energy demand and incremental energy prices. Therefore, the binary variable $z_{t, b, l l, w}$ is required in order to adequately account for the demand in the substation. Variable $z_{t, b, l l, w}$ takes value of 1 for all substation steps $b$ required to cover the energy demanded in the substation. The proposed approach is consistent with the traditional methodology where higher prices are charged to higher substation consumptions, following the rules of the marginal pricing setting. This approximation yields piecewise linear functions, which for practical purposes are indistinguishable from the nonlinear models if enough blocks are used. The lower-level objective function (A11) is linear, since DR only depends on the price difference between adjacent load levels. Analogously, constraints (A12) and (A13), including power balance equations as well as DR limits, are modeled as linear expressions. Hence, the lower-level problem (A11)-(A13) is parameterized in terms of the upper-level decision vector in such a way that the lower-level problem is linear and thus convex.

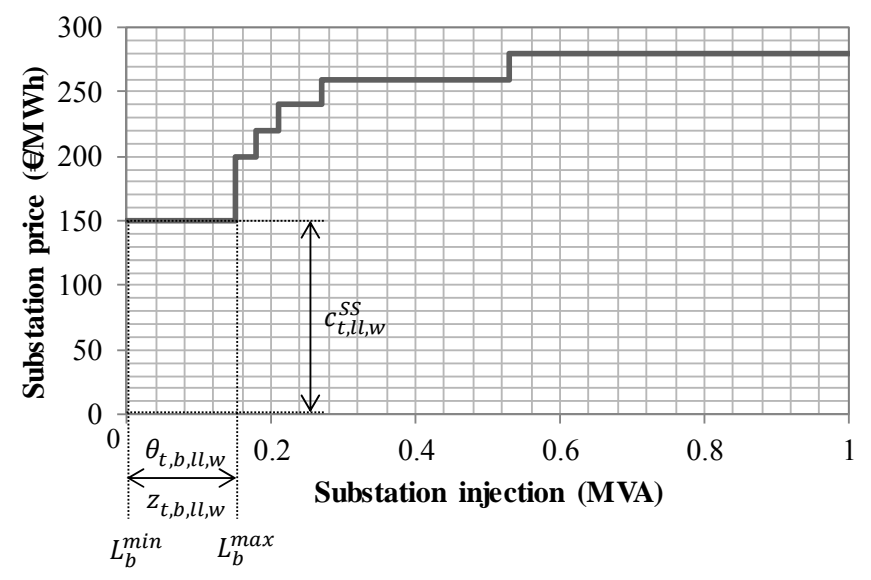

Fig. 3. Substation prices.

Replacing the inner problem of the bi-level problem with the KKT optimality conditions and including the resultant system in the upper-level problem is a well-established technique to solve bi-level problems. Incorporating the lowerlevel problem into the upper one can be achieved either by applying Karush-Kuhn-Tucker (KKT) optimality conditions or by using duality theory. According to [22], both methodologies are equivalent from a rigorous mathematical point of view. The application of KKT conditions yields nonlinearities related to the product of dual and primal variables in the complementarity conditions. For the linearization of such nonlinearities, bounds for dual variables are required, which is customarily set based on experience. The proposed co-optimization avoids the weaknesses associated with the functional decoupling of distribution network planning and distributed generation planning that may lead to economic losses and a suboptimal use of resources.

The following sections provide a comprehensive description of the formulation used in both upper and lower levels. 


\section{A. Upper-Level Objective Function and Constraints}

As previously described, the total cost in (1) consists of four terms: (2) investment, (3) maintenance, (4) energy purchased from substations and DG production and (5) unserved power.

$$
\begin{aligned}
& \text { cost }=\min \left\{\sum_{t} \frac{(1+I)^{-(t-1)}}{I} c_{t}^{I}+\sum_{t}\left[( 1 + I ) ^ { - ( t - 1 ) } \left(c_{t}^{M}+\right.\right.\right. \\
& \left.\left.\left.c_{t}^{E}+c_{t}^{U}\right)\right]+\frac{(1+I)^{-\left(n_{T}-1\right)}}{I}\left(c_{n_{T}}^{M}+c_{n_{T}}^{E}+c_{n_{T}}^{U}\right)\right\}
\end{aligned}
$$

where:

$c_{t}^{I}=\sum_{l \in\{N R F, N A F\}} R R^{l} \sum_{(i, j) \in Y^{l}} C_{i, j}^{I, l} e_{i, j} x_{i, j, t}^{l}+$

$R R^{S S} \sum_{i \in \Omega^{S S}} C_{i}^{I, S S} x_{i, t}^{S S}+R R^{N T} \sum_{i \in \Omega^{S S}} C_{i}^{I, N T} x_{i, t}^{N T}+$

$\sum_{p \in P} R R^{p} \sum_{i \in \Omega^{p}} C_{i}^{I, p} p f \bar{G}^{p} x_{i, t}^{p} ; \quad \forall t \in T$

$c_{t}^{M}=\sum_{l \in L} \sum_{(i, j) \in \Upsilon^{l}} C_{i, j}^{M, l}\left(y_{i, j, t}^{l}+y_{j, i, t}^{l}\right)+$

$\sum_{t r \in T R} \sum_{i \in \Omega^{S S}} C_{i}^{M, t r} y_{i, t}^{t r}+\sum_{p \in P} \sum_{i \in \Omega^{p}} C_{i}^{M, p} y_{i, t}^{p} ; \quad \forall t \in T$

$c_{t}^{E}=\sum_{w \in \Pi} \sum_{l l \in L L} \pi_{l l, w} p f \Delta_{l l}\left(\sum_{t r \in T R} \sum_{i \in \Omega} S S c_{t, l l, w}^{S S} g_{i, t, l l, w}^{t r}\right.$

$\left.+\sum_{p \in P} \sum_{i \in \Omega^{p}} C^{E, p} g_{i, t, l l, w}^{p}\right) ; \forall t \in T$

$c_{t}^{U}=\sum_{\omega \in \Pi} \sum_{l l \in L L} \pi_{l l, w} p f \Delta_{l l} \sum_{i \in \Omega_{t}^{L N}} C^{U} d_{i, t, l l, w}^{U} ; \quad \forall t \in T$

Constraint (6) represents the nodal current balance equations, i.e., Kirchhoff's current law. Fulfillment of Kirchhoff's voltage law is secured by constraint (7). The nonlinear constraint reformulation is presented in Appendix section A. Upper and lower limits for nodal voltages are defined by constraint (8). Maximum capacity of the feeders' constraints current flow are formulated in (9). Following the description provided in [23] and successfully implemented in [1], the linearized network model is an adapted version of the dc model that is based on two assumptions: (i) all current injections and flows have the same power factor, and (ii) the per-unit voltage drop across a branch is equal to the difference between the per-unit magnitudes of the nodal voltages at both ends of the branch. Assumption (i) allows expressing Kirchhoff's current law as a set of linear scalar equalities in terms of current magnitudes. In addition, assumption (ii) allows formulating Kirchhoff's voltage law for branches in use as a linear expression relating the magnitudes of currents, nodal voltages, and branch impedances.

$$
\begin{aligned}
& \sum_{l \in L} \sum_{j \in \Omega_{i}^{l}}\left(f_{i, j, t, l l, w}^{l}-f_{j, i, t, l l, w}^{l}\right)= \\
& \sum_{t r \in T R} g_{i, t, l l, w}^{t r}+\sum_{p \in P} g_{i, t, l l, w}^{p}-d_{i, t, l l, w}+d_{i, t, l l, w}^{U} ; \\
& \forall i \in \Omega^{N}, \forall t \in T, \forall l l \in L L, \forall w \in \Pi \\
& y_{i, j, t}^{l}\left[Z_{i, j}^{l} \ell_{i, j} f_{i, j, t, l l, w}^{l}-\left(v_{i, t, l l, w}-v_{j, t, l l, w}\right)\right]=0 ; \\
& \forall l \in L, \forall i \in \Omega_{j}^{l}, \forall j \in \Omega^{N}, \forall t \in T, \forall l \in L L, \forall \omega \in \Pi \\
& \underline{V} \leq V_{i, t, l l, w} \leq \bar{V} ; \forall i \in \Omega^{N}, \forall t \in T, \forall l \in L L, \forall \omega \in \Pi \\
& 0 \leq f_{i, j, t, l l, w}^{l} \leq y_{i, j, t}^{l} \bar{F}_{i, j}^{l} ; \\
& \forall l \in L, \forall i \in \Omega_{j}^{l}, \forall j \in \Omega^{N}, \forall t \in T, \forall l \in L L, \forall \omega \in \Pi
\end{aligned}
$$

The upper bound for the current that can be supplied by the transformers in use is defined by constraint (10). Renewable generators' outputs are limited by the maximum power availability depending on the technology and the meteorology
(11). Doing so, renewable generators may be curtailed by the operator. Unserved power, $d_{i, t, l l, w}^{U}$, is defined as continuous and non-negative (12). Constraint (13) limits the level of penetration of DG as a fraction of the demand.

$0 \leq g_{i, t, l l, w}^{\text {tr }} \leq y_{i, t}^{\text {tr }} \bar{G}_{i}^{t r}$

$\forall t r \in T R, \forall i \in \Omega^{S S}, \forall t \in T, \forall l l \in L L, \forall w \in \Pi$

$0 \leq g_{i, t, l l, w}^{p} \leq y_{i, t}^{p} \hat{G}_{i, t, l l, w}^{p}$;

$\forall p \in P, \forall i \in \Omega^{p}, \forall t \in T, \forall l l \in L L, \forall \omega \in \Pi$

$0 \leq d_{i, t, l l, w}^{U} \leq d_{i, t, l l, w}$;

$\forall i \in \Omega_{j}^{l}, \forall t \in T, \forall l l \in L L, \forall \omega \in \Pi$

$\sum_{p \in P} \sum_{i \in \Omega^{p}} g_{i, t, l l, w}^{p} \leq \varepsilon \sum_{i \in \Omega_{t}^{L N}} d_{i, t, l l, w} ;$

$\forall t \in T, \forall l l \in L L, \forall w \in \Pi$

Feeders' use is characterized by constraints (14)-(16), explicitly considering the direction of current flows. The utilization of new transformers is formulated in (17) and the use of installed generators is modeled in (18). Only one investment is allowed for each component along the planning horizon (19)-(22). Constraints (23) guarantee that new transformers can only be added at substations that have been previously expanded or built. The overall budget available for investment at each stage is limited by constraint (24).

$y_{i, j, t}^{E F F}+y_{j, i, t}^{E F F}=1 ; \quad \forall(i, j) \in \Upsilon^{E F F}, \forall t \in T$

$y_{i, j, t}^{l}+y_{j, i, t}^{l}=\sum_{\tau=1}^{t} x_{i, j, \tau}^{l} ; \quad \forall l \in$

$\{N R F, N A F\}, \forall(i, j) \in Y^{l}, \forall t \in T$

$y_{i, j, t}^{E R F}+y_{j, i, t}^{E R F}=1-\sum_{\tau=1}^{t} x_{i, j, \tau}^{N R F} ; \forall(i, j) \in \Upsilon^{E R F}, \forall t \in T$

$y_{i, t}^{N T} \leq \sum_{\tau=1}^{t} x_{i, \tau}^{N T} ; \quad \forall i \in \Omega^{S S}, \forall t \in T$

$y_{i, t}^{p} \leq \sum_{\tau=1}^{t} x_{i, \tau}^{p} ; \quad \forall i \in \Omega^{p}, \forall t \in T$

$\sum_{t \in T} x_{i, j, t}^{l} \leq 1 ; \quad \forall l \in\{N R F, N A F\}, \forall(i, j) \in \Upsilon^{l}$

$\sum_{t \in T} x_{i, t}^{S S} \leq 1 ; \quad \forall i \in \Omega^{S S}$

$\sum_{t \in T} x_{i, t}^{N T} \leq 1 ; \quad \forall i \in \Omega^{S S}$

$\sum_{t \in T} x_{i, t}^{p} \leq 1 ; \quad \forall p \in P, \forall i \in \Omega^{p}$

$x_{i, t}^{N T} \leq \sum_{\tau=1}^{t} x_{i, \tau}^{S S} ; \quad \forall i \in \Omega^{S S}, \forall t \in T$

$\sum_{l \in\{N R F, N A F\}} \sum_{(i, j) \in Y^{l}} C_{i, j}^{I, l} \ell_{i j} x_{i, j, t}^{l}+\sum_{i \in \Omega^{S S}} C_{i}^{I, S S} x_{i t}^{S S}+$

$\sum_{i \in \Omega^{S S}} C_{i}^{I, N T} x_{i, t}^{N T}+\sum_{p \in P} \sum_{i \in \Omega^{p}} C_{i}^{I, p} p f \bar{G}^{p} x_{i, t}^{p}+$

$\sum_{p \in P} \sum_{i \in \Omega^{S T}} C_{i}^{I, S T} p f \bar{G}^{S T} x_{i, t}^{S T} \leq I B_{t} ; \quad \forall t \in T$

Radial operation of the distribution networks is guaranteed by constraints (25)-(33). Constraint (25) imposes nodes to have a single input flow while expression (26) sets a maximum of one input flow for the remaining nodes. Authors in [24] have demonstrated that traditional radiality constraints (25)-(26) may fail to guarantee a radial operation of the distribution system when DG is considered. Following the approach presented in [24], a set of radiality constraints (27)-(33) has been added where a fictitious system with fictitious demands is modeled through (27)-(33) in order to avoid the existence of areas exclusively supplied by DG and thereby topologically disconnected from all substations. Fictitious demand at load 
nodes that are candidate locations for DG installation is equal to 1 p.u., being 0 at the remaining nodes (34). These fictitious nodal demands can only be supplied by fictitious substations located at the original substation nodes which inject fictitious energy through the actual feeders. Constraints in (27) represent the fictitious current nodal balance equations. Additionally, constraints (28)-(33) limit the fictitious flows through the fictitious feeders and substations. Constraints (28)-(32) bound the fictitious flows through feeders. Finally, constraints (33) set the limits for the fictitious currents injected by the fictitious substations.

$$
\begin{aligned}
& \sum_{l \in L} \sum_{i \in \Omega_{j}^{l}} y_{i, j, t}^{l}=1 ; \quad \forall j \in \Omega_{t}^{L N}, \forall t \in T \\
& \sum_{l \in L} \sum_{i \in \Omega_{j}^{l}} y_{i, j, t}^{l} \leq 1 ; \quad \forall j \notin \Omega_{t}^{L N}, \forall t \in T \\
& \sum_{l \in L} \sum_{j \in \Omega_{i}^{l}}\left(\tilde{f}_{i, j, t}^{l}-\tilde{f}_{j, i, t}^{l}\right)=\tilde{g}_{i, t}^{S S}-\widetilde{D}_{i, t} ; \forall i \in \Omega^{N}, \forall t \in T \\
& 0 \leq \tilde{f}_{i, j, t}^{E F F} \leq n_{D G} ; \quad \forall i \in \Omega_{j}^{E F F}, \forall j \in \Omega^{N}, \forall t \in T \\
& 0 \leq \tilde{f}_{i, j, t}^{E R F} \leq n_{D G}\left(1-\sum_{\tau=1}^{t} x_{i j \tau}^{N R F}\right) ; \forall(i, j) \in \Upsilon^{E R F}, \forall t \in T \\
& 0 \leq \tilde{f}_{j, i, t}^{E R F} \leq n_{D G}\left(1-\sum_{\tau=1}^{t} x_{i j \tau}^{N R F}\right) ; \forall(i, j) \in \Upsilon^{E R F}, \forall t \in T \\
& 0 \leq \tilde{f}_{i, j, t}^{l} \leq n_{D G} \sum_{\tau=1}^{t} x_{i, j, \tau}^{l} ; \\
& \forall l \in\{N R F, N A F\}, \forall(i, j) \in \Upsilon^{l}, \forall t \in T \\
& 0 \leq \tilde{f}_{j, i, t}^{l} \leq n_{D G} \sum_{\tau=1}^{t} x_{i, j, \tau}^{l} ; \\
& \forall l \in\{N R F, N A F\}, \forall(i, j) \in \Upsilon^{l}, \forall t \in T \\
& 0 \leq \tilde{g}_{i, t}^{S S} \leq n_{D G} ; \quad \forall i \in \Omega^{S S}, \forall t \in T \\
& \widetilde{D}_{i, t}=\left\{\begin{array}{l}
1 ; \forall i \in\left(\left(\Omega^{W} \cup \Omega^{\Theta}\right) \cap \Omega_{t}^{L N}\right), \forall t \in T \\
0 ; \quad \forall i \notin\left(\left(\Omega^{W} \cup \Omega^{\Theta}\right) \cap \Omega_{t}^{L N}\right), \forall t \in T
\end{array}\right.
\end{aligned}
$$

To adequately integrate price-dependent resources, the substation price has been defined as a function of the injected power in the distribution network (see Fig. 3). Equations (1), (4), (35) and (36) are affected by these variable substation costs. Substation prices are variables of the upper level.

The upper level determines the substation prices, which are the linkage between both levels. Based on substation prices, (35) and (36) define the maximum and minimum amount of shiftable load for every load level. As can be seen (35) and (36) are nonlinear expressions which can be linearized as explained in Appendix section B.

$d r_{i, t, l l, w}^{+}=$
$\max \left\{0, \sum_{l b} \xi_{l b, l l} \frac{\left(D_{i, t, l l, w} \Delta_{l l}+D_{i, t, l b, w} \Delta_{l b}\right)}{\Delta_{l b}+\Delta_{l l}} \frac{\left(c_{t, l b, w}^{S S}-\overline{C_{t, l l, w}^{S S}}\right)}{\overline{C_{t, l l, w}^{S S}}}\right\}$

$\forall i \in \Omega^{L N}, \forall t \in T, \forall l l \in L L, \forall w \in \Pi$

$d r_{i, l l, w}^{-}=$

$\max \left\{0, \sum_{l b} \xi_{l b, l l} \frac{\left(D_{i, t, l l, w} \Delta_{l l}+D_{i, t, l b, w} \Delta_{l b}\right)}{\Delta_{l b}+\Delta_{l l}} \frac{\left(c_{t, l l, w}^{S S}-\overline{C_{t, l l, w}^{S S}}\right)}{\overline{C_{t, l l, w}^{S S}}}\right\}$

$\forall i \in \Omega^{L N}, \forall t \in T, \forall l l \in L L, \forall w \in \Pi$

$\overline{C_{t, l l, w}^{S S}}=\frac{\sum_{l b} c_{t, l b, w}^{S S} \Delta_{l b}}{\sum_{l b} \Delta_{l b}} \quad \forall t \in T, \forall l l \in L L, \forall w \in \Pi$

The aforementioned equation (4) models all production costs including substation production costs, which are dependent on the substation price for the considered load level and generation of the individual units. To do this, a binary expansion approach [16] has been used. The discrete substation price, $c_{t, l l, w}^{S S}$, has been defined by $b$ steps (or blocks) between
$L_{b}{ }^{\min }$ and $L_{b}{ }^{\text {max }}$ by adding binary variable $z_{t, b, l l, w}$ (38). Variable $\theta_{t, b, l l, w}$ represents the level of substation power injection for each considered step $b$ (Fig. 3). The value of $\theta_{t, b, l l, w}$ is limited by the upper and lower limits of the considered step (39). The sequentiality of the active step $b$ introduced in the formulation of the substation price is guaranteed in (40). Equation (41) guarantees the equivalence between the sum of the energy supplied by the different transformers and the considered power injection. The nonlinear constraint reformulation is presented in Appendix section C. These constraints are included in the upper-level formulation.

$c_{t, l l, w}^{S S}=\sum_{b \in \Omega^{B}} C_{b} z_{t, b, l l, w} \quad \forall t \in T, \forall l l \in L L, \forall w \in \Pi$

$z_{t, b, l l, w} L_{b}{ }^{\min } \leq \theta_{t, b, l l, w} \leq z_{t, b, l l, w} L_{b}{ }^{\max }$

$\forall b \in B, \forall t \in T, \forall l l \in L L, \forall w \in \Pi$

$z_{t, b, l l, w} \geq z_{t, b+1, l l, w}$

$\forall b \in B, \forall t \in T, \forall l l \in L L, \forall w \in \Pi$

$\sum_{i \in \Omega} s s g_{i, t, l l, w}^{t r}=\sum_{b \in \Omega^{B}} Z_{t, b, l l, w} \theta_{t, b, l l, w}$

$\forall t \in T, \forall l l \in L L, \forall w \in \Pi$

\section{B. Lower-Level Objective Function and Constraints}

The objective function of the lower level represents the total payment that the consumers have to afford over the considered time horizon. DR (42) has been introduced in the model considering elastic demand functions calibrated by load levels. Final demand levels are defined in (43). Constraint (44) guarantees that the overall positive and negative demand responses are equivalent. DR load shifting is limited by the maximum amount of shiftable load (defined as a function of the upper and lower limits $d r_{i, t, l l, w}^{+}$and $d r_{i, t, l l, w}^{-}$), expressed as a function of the substation prices and the demanded energy, which is a parameter at this level (45)-(46). Lower-level variables are the final demand $\left(d_{i, t, l l, w}\right)$ and demand modifications $\left(d r_{i, t, l l, w}\right)$.

$$
\begin{aligned}
& \text { payment }=\min \left\{\sum _ { t , l l , w } \left((1+I)^{-(t-1)}+\right.\right. \\
& \left.\left.\frac{(1+I)^{-\left(n_{T^{-1}}\right)}}{I}\right)\left[\pi_{l l, w} p_{t, l l, w}\right]\right\}
\end{aligned}
$$

subject to:

$$
d_{i, t, l l, w}=D_{i, t, l l, w}+d r_{i, t, l l, w}: \lambda^{1}{ }_{i, t, l l, w}
$$$$
\forall i \in \Omega^{L N}, \forall t \in T, \forall l l \in L L, \forall w \in \Pi
$$

$\sum_{l l} d r_{i, t, l l, w} * \Delta_{l l}=0: \lambda^{2}{ }_{i, t, w}$

$\forall i \in \Omega^{L N}, \forall t \in T, \forall w \in \Pi$

$d r_{i, t, l l, w}^{+}-d r_{i, t, l l, w} \geq 0: \mu^{1}{ }_{i, t, l l, w}$

$\forall i \in \Omega^{L N}, \forall t \in T, \forall l l \in L L, \forall w \in \Pi$

$d r_{i, t, l l, w}+d r_{i, t, l l, w}^{-} \geq 0: \mu^{2}{ }_{i, t, l l, w}$

$\forall i \in \Omega^{L N}, \forall t \in T, \forall l l \in L L, \forall w \in \Pi$

The price elasticity assumptions determine the slope of the demand function with own-price elasticities, $\xi_{l l, l l}$, and crossprice elasticities, $\xi_{l b, l l}$, being exogenously provided, based upon values from the literature. Own-price elasticity refers to how energy consumers react to every single load level, considering the average price of the incumbent load levels. Cross-price elasticity refers to the consumer's reaction to the 
prices in other load levels. The addition of price elasticity results in demand function $d_{i, t, l l, w}$, which expresses the quantity demanded, $d_{i, t, l l, w}$, as function of the relative deviations of load level prices from the reference level. For simplicity reasons, both own and cross elasticities are considered to have the same value. Based on [15]-[16], the present paper analyses five different values for cross-price elasticity between day and night periods and between the levels corresponding to the same time block ranging from 0 to $4 \%$. For each quarter $(q)$ and working day/weekend $(r)$, the energy can be exchanged between: blocks of the same day; blocks of the same night; blocks from day to night; blocks from night to day.

Incorporating the KKT conditions (as previously described), the lower-level problem is reformulated as:

$\mu^{1}{ }_{i, t, l l, w} \geq 0 \forall i \in \Omega^{L N}, \forall t \in T, \forall l l \in L L, \forall w \in \Pi$

$\mu^{2}{ }_{i, t, l l, w} \geq 0 \forall i \in \Omega^{L N}, \forall t \in T, \forall l l \in L L, \forall w \in \Pi$

$\sum_{t, b, l l, w}\left((1+I)^{-(t-1)}+\right.$

$\left.\frac{(1+I)^{-\left(n_{T}-1\right)}}{I}\right)\left[\pi_{l l, w} p f C_{b} Z_{t, b, l l, w}\right]-\sum_{i, t, l l, w} \lambda^{1}{ }_{i, t, l l, w}=0$

$\lambda^{1}{ }_{i, t, l l, w}+\mu^{1}{ }_{i, t, l l, w}-\mu^{2}{ }_{i, t, l l, w}-\sum_{l l} \lambda^{2}{ }_{i, t, w} \Delta_{l l}=0$

$\forall i \in \Omega^{L N}, \forall t \in T, \forall l l \in L L, \forall w \in \Pi$

$$
\begin{aligned}
& \left(d r_{i, t, l l, w}^{+}-d r_{i, t, l l, w}\right) \mu^{1}{ }_{i, t, l l, w}=0 \\
& \forall i \in \Omega^{L N}, \forall t \in T, \forall l l \in L L, \forall w \in \Pi \\
& \left(d r_{i, t, l l, w}-d r_{i, t, l l, w}^{-}\right) \mu^{2}{ }_{i, t, l l, w}=0 \\
& \forall i \in \Omega^{L N}, \forall t \in T, \forall l l \in L L, \forall w \in \Pi
\end{aligned}
$$

Electricity customers in isolated electric power systems expect reasonable and non-discriminatory prices. Substation prices have been considered in the proposed methodology as an adequate non-discriminatory price signal for consumers. Equation (53) models the demand payment for each load level as a function of the substation price and the total volume of demand (Fig. 3). Total payment faced by the consumers is included in the objective function of the lower level (42). Due to the intrinsic nature of bilevel programming, overall payment of the consumers and its related constraints are replaced by its KKT conditions. Therefore, overall payment of the consumers can only be calculated ex-post in the proposed approach. Due to the previous considerations, the non-linear expression presented in (53) does not require a specific linearization.

$p_{t, l l, w}=\sum_{i \in \Omega^{N}} c_{t, l l, w}^{S S} p f d_{i, t, l l, w}$

$\forall t \in T, \forall l l \in L L, \forall w \in \Pi$

\section{CASE STUDY}

La Graciosa is a small island belonging to the Canary Islands located near Lanzarote and is connected to the Lanzarote-Fuerteventura electric power system, consisting of 26 nodes and 37 branches. La Graciosa is connected to the Lanzarote - Fuerteventura electric power system. The distribution network analyzed consists of 23 load nodes, 3 substation nodes, and 37 branches (Fig. 4). This section presents the results of the proposed methodology for La Graciosa system, including a comparative analysis based on different elasticities. The results of the proposed expansion- planning algorithm after the considered time horizon are presented in Fig. 6-8. A baseline situation where no elasticity is considered $\left(\xi_{l l, l l}=\xi_{l b, l l}=0 \%\right)$ has been defined in order to adequately present the results. Then, different scenarios increasing the elasticity are defined (ranging from $0 \%$ to $4 \%$ ).

\section{A. System Description}

The planning horizon is 15 years subdivided into five periods of three years, following the approach proposed in [1]. For reproducibility purposes, data for nodal peak demands and branch lengths as well as the single line diagram for the considered system can be downloaded from [25].

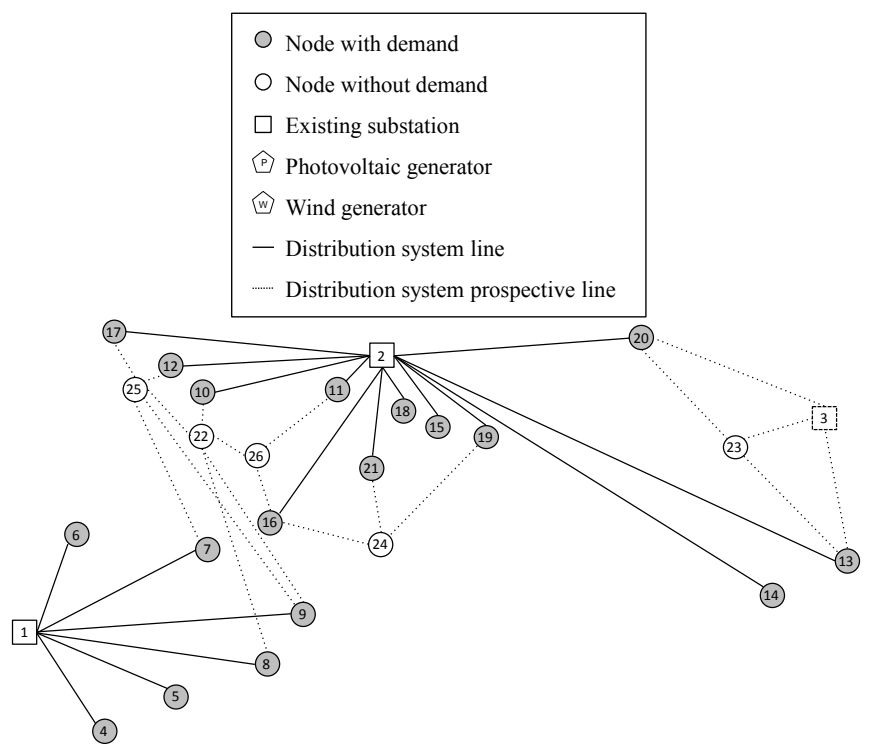

Fig. 4. One-line diagram of the La Graciosa distribution network.

\section{B. Results}

Results presented in Tables II and III outline the impact of DR in the expansion planning problem, reducing costs for the generation and distribution company and reducing the overall

\begin{tabular}{|c|c|c|c|c|c|}
\hline Costs & $\xi_{l b, l l} 0 \%$ & $\xi_{l b, l l} 1 \%$ & $\xi_{l b, l l} 2 \%$ & $\xi_{l b, l l} 3 \%$ & $\xi_{l b, l l} 4 \%$ \\
\hline Investment & 346.37 & 333.20 & 333.20 & 350.00 & 350.00 \\
\hline O\&M & 330.30 & 322.64 & 322.64 & 338.57 & 338.57 \\
\hline $\begin{array}{c}\text { Energy } \\
\text { purchased }\end{array}$ & 5959.75 & 5863.06 & 5796.49 & 5646.86 & 5538.90 \\
\hline $\begin{array}{c}\text { Unserved } \\
\text { power }\end{array}$ & 0.00 & 0.00 & 0.00 & 0.00 & 0.00 \\
\hline Total & 6636.42 & 6518.91 & 6452.34 & 6335.43 & 6227.47 \\
\hline \multicolumn{6}{|c|}{ TABLE II } \\
\hline & $\xi_{l b, l l} 0 \%$ & $\xi_{l b, l l} 1 \%$ & $\xi_{l b, l l} 2 \%$ & $\xi_{l b, l l} 3 \%$ & $\xi_{l b, l l} 4 \%$ \\
\hline Payment & 7967.84 & 7892.81 & 7883.27 & 7785.43 & 7721.04 \\
\hline
\end{tabular}
payment for the consumers.

TABLE II

TOTAL OPERATING AND INVESTMENT COSTS [THOUSANDS OF $€$ ]

It can be noticed how a higher elasticity benefits both central planner and consumers, reducing the operating and investment costs and the payment from the consumers. Additionally, operating and investment costs reduction are bigger than the demand payment reduction, showing the potential benefit derived from the introduction of DR for the central planner under the considered framework. The long- 
term benefit allowing demand to respond to time-varying price conditions is a flatter load shape, which should reduce the need for peak saving capacity and, in turn, reduce emissions and costs through the more effective and efficient use of the grid. Both generation and distribution companies and final consumers are benefited in long-term planning from the exposure of consumers to real-time prices. In order to adequately integrate the short-term reaction of the consumers in the short term into the long-term expansion planning proposed, and aiming at achieving the higher economic benefit for the consumers derived from its flexibility, the traditional formulation has been relaxed replacing the equality constraint by the definition of a maximum value, as presented in Eq. (35)-(37), where DR load shifting is limited by the maximum amount of shiftable load, expressed as a function of the substation prices and the demanded energy. In order to adequately integrate the short-term reaction of the consumers into the long-term expansion planning proposed, and aiming at achieving the higher economic benefit for the consumers derived from its flexibility, variables $d r_{i, t, l l, w}^{+}$and $d r_{i, t, l l, w}^{-}$only set the limits for the variation of demand, while demand can be freely modified in between them to suit the interests of the consumers. Therefore, the inherent flexibility of the consumers results in lower payment of the demand and higher costs for the generation and distribution company when the bi-level approach is proposed, as presented in Tables IV and V. For the same reason, the single-level formulation allows the generation and distribution planner to take advantage of this flexibility, resulting in lower operating and investment costs, as presented in Table V. In order to better understand the comparison presented in Table IV, a counterpart of Table II for the singlelevel formulation is presented in Table VI. Increasing the flexibility of the consumers result in a reduction of the total payment, since consumers would act driven by economic incentives taking advantage of their flexibility to reduce their payment. On the other hand, from the presented results it can be noticed how a higher elasticity benefits both central planner and consumers, reducing the operating and investment costs and the payment from the consumers.

TABLE IV

TOTAL OPERATING AND INVESTMENT COSTS [THOUSANDS OF $€$ ]

\begin{tabular}{cccccc} 
& $\xi_{l b, l l} 0 \%$ & $\xi_{l b, l l} 1 \%$ & $\xi_{l b, l l} 2 \%$ & $\xi_{l b, l l} 3 \%$ & $\xi_{l b, l l} 4 \%$ \\
\hline Bi-level & 6636.42 & 6518.91 & 6452.34 & 6335.43 & 6227.47 \\
\hline Single-level & 6636.42 & 6502.65 & 6411.93 & 6269.82 & 6157.66 \\
\hline
\end{tabular}

TABLE V

TOTAL PAYMENT OF THE DEMAND [THOUSANDS OF $€$ ]

\begin{tabular}{cccccc} 
& $\xi_{l b, l l} 0 \%$ & $\xi_{l b, l l} 1 \%$ & $\xi_{l b, l l} 2 \%$ & $\xi_{l b, l l} 3 \%$ & $\xi_{l b, l l} 4 \%$ \\
\hline Bi-level & 7967.84 & 7892.81 & 7883.27 & 7785.43 & 7721.04 \\
\hline Single-level & 7967.84 & 7898.15 & 7889.04 & 7812.61 & 7760.95 \\
\hline
\end{tabular}

TABLE VI

SINGLE-LEVEL TOTAL OPERATING AND INVESTMENT COSTS [THOUSANDS OF $€$ ]

\begin{tabular}{cccccc}
\hline Costs & $\boldsymbol{\xi}_{\boldsymbol{l b}, \boldsymbol{l l}} \mathbf{0} \%$ & $\boldsymbol{\xi}_{\boldsymbol{l b}, \boldsymbol{l l}} \mathbf{1} \%$ & $\boldsymbol{\xi}_{\boldsymbol{l b}, \boldsymbol{l l}} \mathbf{2} \%$ & $\boldsymbol{\xi}_{\boldsymbol{l b}, \boldsymbol{l l}} \mathbf{3} \%$ & $\boldsymbol{\xi}_{\boldsymbol{l b}, \boldsymbol{l l}} \mathbf{4} \%$ \\
\hline Investment & 346.37 & 346.37 & 328.10 & 333.20 & 341.60 \\
\hline O\&M & 330.30 & 330.30 & 326.42 & 322.64 & 334.63 \\
\hline $\begin{array}{c}\text { Energy } \\
\text { purchased }\end{array}$ & 5959.75 & 5825.98 & 5757.41 & 5613.98 & 5481.43 \\
\hline $\begin{array}{c}\text { Unserved } \\
\text { power }\end{array}$ & 0 & 0 & 0 & 0 & 0 \\
\hline Total & 6636.42 & 6502.65 & 6411.93 & 6269.82 & 6157.66 \\
\hline
\end{tabular}

Tables VII and VIII present the investment decisions for wind and PV technologies. Analyzing Tables VII and VIII, it is worth mentioning how DR modifies the deployment of renewable technologies in the distribution network.

TABLE VII

WIND GENERATION INVESTMENT DECISIONS PER STAGE

\begin{tabular}{cccccc}
\hline Stage & $\boldsymbol{\xi}_{\boldsymbol{l b}, \boldsymbol{l l}} \mathbf{0} \%$ & $\boldsymbol{\xi}_{\boldsymbol{l b}, \boldsymbol{l l}} \mathbf{1} \%$ & $\boldsymbol{\xi}_{\boldsymbol{l b}, \boldsymbol{l l}} \mathbf{2} \%$ & $\boldsymbol{\xi}_{\boldsymbol{l b}, \boldsymbol{l l}} \mathbf{3} \%$ & $\boldsymbol{\xi}_{\boldsymbol{l b}, \boldsymbol{l} \boldsymbol{l}} \mathbf{4} \%$ \\
\hline 1 & $\mathrm{~N} 8, \mathrm{~N} 16$ & $\mathrm{~N} 8, \mathrm{~N} 16$ & $\mathrm{~N} 8, \mathrm{~N} 16$ & $\mathrm{~N} 8, \mathrm{~N} 16$ & $\mathrm{~N} 8, \mathrm{~N} 16$ \\
\hline 2 & $\mathrm{~N} 7, \mathrm{~N} 13$ & $\mathrm{~N} 13$ & $\mathrm{~N} 13$ & $\mathrm{~N} 7, \mathrm{~N} 13$ & $\mathrm{~N} 7, \mathrm{~N} 13$ \\
\hline 3 & $\mathrm{~N} 10$ & $\mathrm{~N} 10$ & & $\mathrm{~N} 10$ \\
\hline 4 & $\mathrm{~N} 10$ & $\mathrm{~N} 7$ & $\mathrm{~N} 7$ & $\mathrm{~N} 10$ & $\mathrm{~N} 11$ \\
\hline 5 & & $\mathrm{~N} 11$ & $\mathrm{~N} 11$ & $\mathrm{~N} 11$ &
\end{tabular}

Photovoltaic GENERATION INVESTMENT DECISIONS PER STAGE

\begin{tabular}{cccccc}
\hline Stage & $\boldsymbol{\xi}_{l b, l l} \mathbf{0} \%$ & $\boldsymbol{\xi}_{l b, l l} \mathbf{1} \%$ & $\boldsymbol{\xi}_{l b, l l} \mathbf{2} \%$ & $\boldsymbol{\xi}_{l b, l l} \mathbf{3} \%$ & $\boldsymbol{\xi}_{l b, l l} \mathbf{4} \%$ \\
\hline 1 & \multicolumn{5}{c}{} \\
\hline 2 & $\mathrm{~N} 14$ & $\mathrm{~N} 14$ & & \\
\hline 3 & $\begin{array}{c}\mathrm{N} 4, \mathrm{~N} 8, \\
\mathrm{~N} 14\end{array}$ & $\mathrm{~N} 4$ & $\mathrm{~N} 4$ & $\begin{array}{c}\mathrm{N} 4, \mathrm{~N} 8, \\
\mathrm{~N} 14\end{array}$ & $\begin{array}{c}\text { N4,N8, } \\
\text { N14 }\end{array}$ \\
\hline 4 & $\mathrm{~N} 13$ & $\mathrm{~N} 8$ & $\mathrm{~N} 8$ & $\mathrm{~N} 13$ & $\mathrm{~N} 13$ \\
\hline 5 & $\mathrm{~N} 12, \mathrm{~N} 16$ & $\mathrm{~N} 16$ & $\mathrm{~N} 16$ & $\mathrm{~N} 16$ & $\mathrm{~N} 16$ \\
\hline
\end{tabular}

A major advantage of DR is its potential for deferring investments in distribution network capacity driven by natural demand growth. Results presented in Table IX outline this effect, leading to a deferment in network investment when increasing the elasticity. In Table IX, the nomenclature n-n represents replaced feeders, while N-N represents added feeders.

\begin{tabular}{|c|c|c|c|c|c|}
\hline Stage & $\xi_{l b, l l} \mathbf{0} \%$ & $\xi_{l b, l l} 1 \%$ & $\xi_{l b, l l} 2 \%$ & $\xi_{l b, l l} 3 \%$ & $\xi_{l b, l l} 4 \%$ \\
\hline 1 & $\begin{array}{c}\mathrm{n} 1-\mathrm{n} 8, \\
\mathrm{~N} 3-\mathrm{N} 23, \\
\mathrm{~N} 8-\mathrm{N} 22, \\
\mathrm{~N} 22-\mathrm{N} 25\end{array}$ & $\begin{array}{c}\mathrm{n} 1-\mathrm{n} 8, \\
\mathrm{~N} 3-\mathrm{N} 23, \\
\mathrm{~N} 8-\mathrm{N} 22, \\
\mathrm{~N} 22-\mathrm{N} 25\end{array}$ & $\begin{array}{c}\mathrm{n} 1-\mathrm{n} 8, \\
\mathrm{~N} 3-\mathrm{N} 23, \\
\mathrm{~N} 8-\mathrm{N} 22, \\
\mathrm{~N} 22-\mathrm{N} 25\end{array}$ & $\begin{array}{l}\text { n2-n16, } \\
\text { N3-N23, } \\
\text { N8-N22 }\end{array}$ & $\begin{array}{l}\text { N3-N23, } \\
\text { N8-N22 }\end{array}$ \\
\hline 2 & & & & $\begin{array}{l}\text { N7-N25, } \\
\text { N16-N26 }\end{array}$ & N7-N25 \\
\hline 3 & $\begin{array}{l}\text { N16-N24, } \\
\text { N16-N26 }\end{array}$ & N16-N26 & N16-N26 & & N16-N26 \\
\hline 4 & & N16-N24 & N16-N24 & N16-N24 & N16-N24 \\
\hline
\end{tabular}

The final situation of the distribution system of La Graciosa is presented in Figs. 5-7, showing the expansion of the distribution network to cover the expected demand increase and the installation of distributed generation within the distribution network for different levels of elasticity.

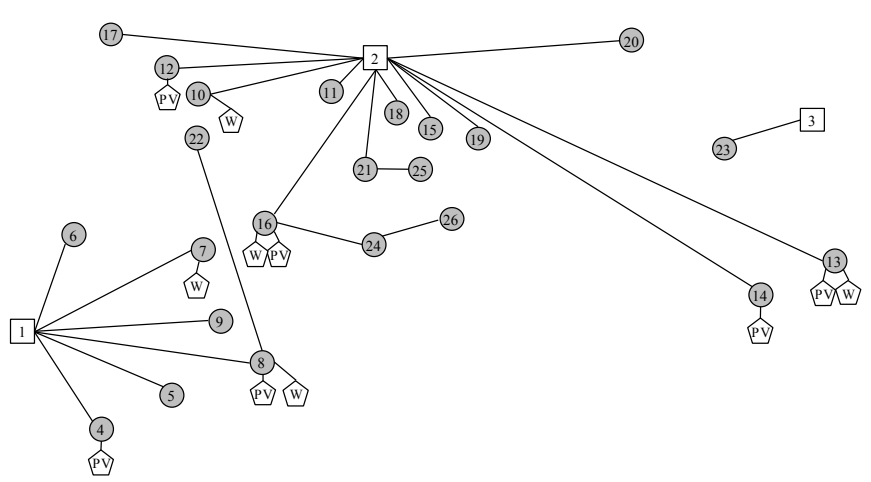

Fig. 5. One-line diagram of the La Graciosa distribution network with $0 \%$ elasticity. 
It must be noted how the integration of demand response programs allowing consumers to respond to electricity prices and adapting their electricity consumption accordingly impacts the generation and distribution network expansion planning.

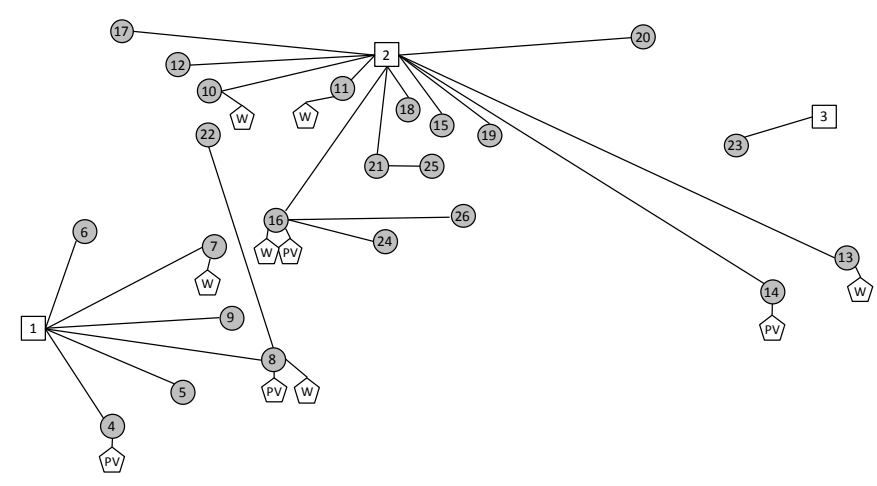

Fig. 6. One-line diagram of the La Graciosa distribution network with $2 \%$ elasticity.

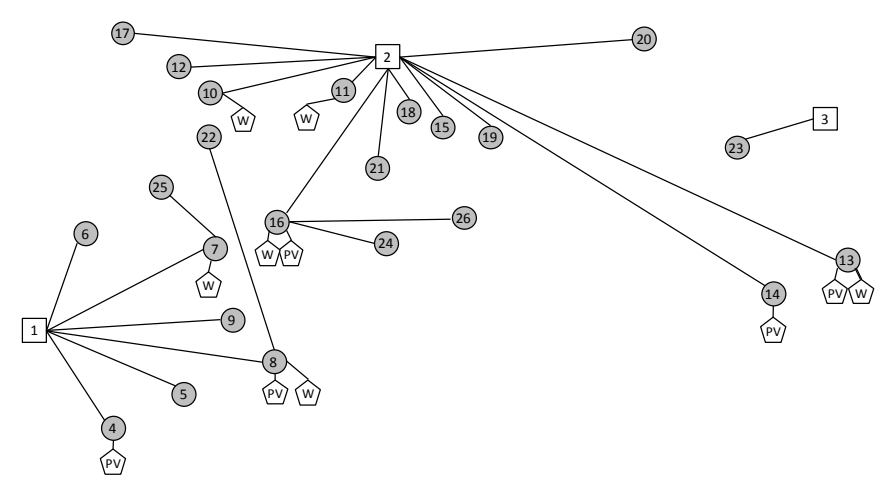

Fig. 7. One-line diagram of the La Graciosa distribution network with $4 \%$ elasticity.

Simulations have been run on a Dell PowerEdge R910X64 with 4 Intel Xeon E7-4820 processors at $2 \mathrm{GHz}$ and $768 \mathrm{~GB}$ of RAM using CPLEX 12.6 [26] under GAMS 24.2.3 [27]. The stopping criterion for the branch-and-cut algorithm of CPLEX used in the proposed model is based on an optimality gap equal to $0.5 \%$. Under this stopping criterion, the attainment of each solution took $10 \mathrm{~h}$ on average. The results indicate the practical applicability of the proposed long-term distribution network expansion planning algorithm, especially considering the time frame of the problem addressed. The computational time is highly dependent on the optimality gap to ensure accuracy of the solution and the number of binary variables which are dependent on the considered network (replaceable lines and location of technologies). For bigger systems, the number of replaceable lines and location of new technologies can be reduced or fixed (which is a reasonable assumption) in order to make the problem tractable.

\section{COnClusions}

DR is a promising subject in operation and planning of electrical power systems. However, its impact in joint generation and distribution expansion planning has not been fully analysed yet. Its relevance in short-term operation of power systems has been extensively investigated, where symmetric treatment of demand and generation creates the strongest incentive for final consumers to participate actively in the wholesale electricity market. When considering medium- and long-term planning, demand responsiveness has an impact on reduction or deferment in distribution expansion planning. The present paper demonstrates how DR can contribute to adequately accommodate renewable generation in a joint expansion planning. Additionally, the potential of DR for deferring investments has been successfully demonstrated.

\section{APPENDIX. MODEL LINEARIZATION}

The optimization model for the joint generation and distribution expansion planning presented includes nonlinearities, which make the optimal solution hard to obtain. Instead of directly addressing the original problem of mixedinteger nonlinear programming, a mixed-integer linear formulation is proposed. These nonlinearities are recast as linear expressions by using a piecewise linear approximation and integer algebra results for the bilinear terms.

\section{A. Kirchhoff's Law Linearization}

Constraints in (7) model Kirchhoff's voltage law for all feeders in use. The equivalent integer linear reformulation is defined in (B1), where $M$ is a large enough positive constant and its influence is similar to constraint (7). This non-linearity is modeled through binary variables:

$$
\begin{aligned}
&-M\left(1-y_{i, j, t}^{l}\right) \leq Z_{i, j}^{l} f_{i, j, l l, w}^{l}-\left(v_{i, t, l l, w}-v_{j, t, l l, w}\right) \\
& \leq M\left(1-y_{i, j, t}^{l}\right) ; \\
& \forall l \in L, \forall i \in \Omega_{j}^{l}, \forall j \in \Omega^{N}, \forall t \in T, \forall l \in L L, \forall \omega \in \Pi
\end{aligned}
$$

\section{B. Demand Response Linearization}

Constraints (35) and (36) represent positive and negative demand shifting, respectively. The equivalent integer linear reformulation is defined in (B2)-(B3). Variable $z_{t, l l, w}^{d r}$ is defined as a binary variable associated with DR at stage $t$ for load level $l l$ and scenario $w$. Its formulation is consequent with the standard formulation of the Big-M linearization procedure, as presented by Fortuny-Amat in [28].

$$
\begin{aligned}
& -M\left(1-z_{t, l l, w}^{d r}\right) \leq d r_{i, t, l l, w}^{+}- \\
& \sum_{l b} \xi_{l b, l l} \frac{\left(D_{t, i, l} \Delta_{l l}+D_{t, i, l b} \Delta_{l b}\right)}{\Delta_{l b}+\Delta_{l l}} \frac{\left(c_{t, l b, w}^{S S}-\overline{C_{t, l l, w}^{S S}}\right)}{\overline{C_{t, l l, w}^{S S}}} \quad \leq M(1- \\
& \left.z_{t, l l, w}^{d r}\right) \forall i \in \Omega^{L N}, \forall t \in T, \forall l l \in L L, \forall w \in \Pi \\
& -M z_{t, l l, w}^{d r} \leq d r_{i, t, l l, w}^{-}- \\
& \sum_{l b} \xi_{l b, l l} \frac{\left(D_{t, i, l l} \Delta_{l l}+D_{t, i, l b} \Delta_{l b}\right)}{\Delta_{l b}+\Delta_{l l}} \frac{\left(c_{t, l l, w}^{S S} \overline{C_{t, l l, w}^{S S}}\right)}{\overline{C_{t, l l, w}^{S S}}} \\
& \leq M z_{t, l l, w}^{d r} \forall i \in \Omega^{L N}, \forall t \in T, \forall l l \in L L, \forall w \in \Pi \\
& \text { C. Substation Energy Demand Linearization }
\end{aligned}
$$

The non-linear product of binary and continuous variables defined in (41) can be equivalently reformulated as an integer equation (also known as Big-M reformulation) (B4). In constraint (B4) $M$ is a large-enough positive constant.

$$
\begin{aligned}
& -\left(1-z_{t, b, l l, w}\right) M \leq \theta_{t, b, l l, w}-\sum_{i \in \Omega} s s g_{i, t, l l, w}^{t r} \leq \\
& \left(1-z_{t, b, l l, w}\right) M \quad \forall i \in \Omega^{L N}, \forall t \in T, \forall l l \in L L, \forall w \in \Pi
\end{aligned}
$$




\section{REFERENCES}

[1] G. Muñoz-Delgado, J. Contreras and J. M. Arroyo, "Multistage generation and network expansion planning in distribution systems considering uncertainty and reliability," IEEE Trans. Power Syst., accepted for publication, DOI 10.1109/TPWRS.2015.2503604.

[2] A. Bagheri, H. Monsef and H. Lesani, "Integrated distribution network expansion planning incorporating distributed generation considering uncertainties, reliability, and operational conditions," Int. J. Electr. Power Energy Syst., vol. 73, pp. 56-70, Dec. 2015.

[3] M. Lavorato, M. J. Rider, A. V. Garcia and R. Romero, "A constructive heuristic algorithm for distribution system planning," IEEE Trans. Power Syst., vol. 25, no. 3, pp. 1734-1742, Aug. 2010.

[4] Y. Atwa, E. El-Saadany, M. Salama and R. Seethapathy, "Optimal renewable resources mix for distribution system energy loss minimization," IEEE Trans. Power Syst., vol. 25, no. 1, pp. 360-370, Feb. 2010

[5] J. F. Franco, M. J. Rider and R. Romero "A mixed-integer quadratically-constrained programming model for the distribution system expansion planning," Int. J. Electr. Power Energy Syst., vol. 62, pp. 265-272, Nov. 2014.

[6] H. Shayeghi and A. Bagheri "Dynamic sub-transmission system expansion planning incorporating distributed generation using hybrid DCGA and LP technique," Int. J. Electr. Power Energy Syst., vol. 48, pp. 111-122, Jun. 2013.

[7] Ö. Özdemir, F. D. Munoz, J. L. Ho and B. F. Hobbs, "Economic analysis of transmission expansion planning with pice-responsive demand and quadratic losses by successive LP," IEEE Trans. Smart Grid, vol. 31, no. 2, pp. 1096-1107, Mar. 2016.

[8] C. De Jonghe, B. F. Hobbs and R. Belmans, "Optimal generation mix with short-term demand response and wind penetration," IEEE Trans. Power Syst., vol. 27, no. 2, pp.830-839, May 2012.

[9] I. Ziari, G. Ledwich, A. Ghosh and G. Platt, "Integrated distribution systems planning to improve reliability under load growth," IEEE Trans. Power Syst., vol. 27, no. 2, pp. 757-765, May 2012.

[10] A. Hajebrahimi, A. Abdollahi and M. Rashidinejad, "Probabilistic multiobjective transmission expansion planning incorporating demand response resources and large-scale distant wind farms," IEEE Syst. Journal, accepted for publication, DOI 10.1109/JSYST.2015.2464151.

[11] A. Satchwell and R. Hledik, "Analytical Frameworks to Incorporate Demand Response in Long-Term Resource Planning," Lawrence Berkeley National Laboratory, DE-AC02-05CH11231, Sep. 2013 [Online]. Available: https://emp.lbl.gov/sites/all/files/lbnl-6546e_0.pdf

[12] J. F. Bard, "Practical bilevel optimization. Algorithms and applications," Kluwer Academic Publishers, 1998.

[13] S. Dempe, "Foundations of bilevel programming", Kluwer Academic Publishers, 2002.

[14] J. M. López-Lezama, A. Padilha-Feltrin, J. Contreras and J.I. Muñoz, "Optimal contract pricing of distributed generation in distribution networks," IEEE Trans. Power Syst., 2011, 26, (1), pp. 128-136.

[15] M. G. Lijesen, "The real-time price elasticity of electricity," Energy Econon., vol. 29, no. 2, pp. 249-258, 2007.

[16] K. Dietrich, J. M. Latorre, L. Olmos and A. Ramos, "Demand response in an isolated system with high wind integration," IEEE Trans. Power Syst., vol. 27, no.1 pp. 20-29, Feb. 2012.

[17] J. Contreras, M. Asensio, P. Meneses de Quevedo, G. Muñoz-Delgado and S. Montoya-Bueno, "Joint RES and Distribution Network Expansion Planning under a Demand Response Framework,” 2016, Academic Press, ISBN 978-0128053225.

[18] L. Baringo and A. J. Conejo, "Wind power investment within a market environment," Appl. Energy, vol. 88, no. 9, pp. 3239-3247, Sep. 2011.

[19] S. Montoya-Bueno, J. I. Muñoz, and Javier Contreras, "DG allocation for benefit maximization in distribution networks," IEEE Transactions on Sustainable Energy, vol. 6, no. 4, Oct. 2015

[20] S. Krohn, P. Morthorst, and S. Awerbuch, "The Economics of Wind Energy," EWEA Report online available: http://www.ewea.org/fileadmin/files/library/publications/reports/Econo mics_of_Wind_Energy.pdf, 2009

[21] D. S. Ryberg, J. Freeman, and N. Blair, "Quantifying Interannual Variability for Photovoltaic Systems in PVWatts," NREL, online available: http://www.nrel.gov/docs/fy16osti/64880.pdf, October 2015.

[22] J. M. Arroyo, "Bilevel programming applied to power system vulnerability analysis under multiple contingencies," IET Gener. Transm. Distrib., vol. 4, no. 2, pp. 178-190, 2010.
[23] S. Haffner, L. F. A. Pereira, L. A. Pereira, and L. S. Barreto, "Multistage model for distribution expansion planning with distributed generation- Part I: Problem formulation," IEEE Trans. Power Deliv., vol. 23, no. 2, pp. 915-923, Apr. 2008.

[24] M. Lavorato, J. F. Franco, M. J. Rider and R. Romero, "Imposing radiality constraints in distribution system optimization problems," IEEE Trans. Power Syst., vol. 27, no. 1, pp. 172-180, Feb. 2012.

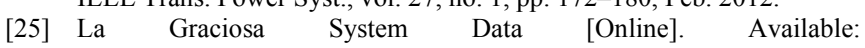
https://drive.google.com/open?id=0B9LAC0eIGvpbNUtOaUwzQktSUl E

[26] The IBM ILOG CPLEX Website. 2016. [Online]. Available: http:// www-01.ibm.com/software/commerce/optimization/cplex-optimizer.

[27] The GAMS Development Corporation Website. 2016. [Online]. Available: http://www.gams.com.

[28] J. Fortuny-Amat and B. McCarl, "A representation and economic interpretation of a two-level programming problem," J. Oper. Res. Soc., vol. 32, no. 9, pp. 783-792, Sep. 1981.

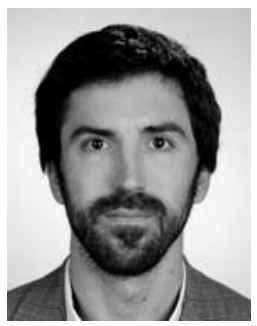

Miguel Asensio received the Ingeniero Industrial degree from the Polytechnic University of Madrid, Spain, in 2003, the MSc degree from Comillas University, Madrid, Spain, in 2013 and the Ph.D. degree from the University of Castilla - Mancha, Spain, in 2016. He has been working in the German, Italian and Spanish Electrical Sector between 2003 and 2012. His research interests lie within the area of decision-making support systems in the energy sector, electricity market and operational issues and transmission pricing.

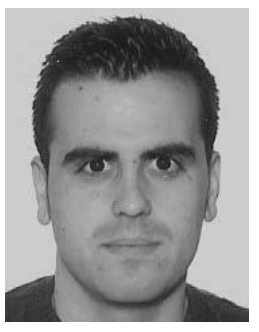

Gregorio Muñoz-Delgado (S'14) received the Ingeniero Industrial degree and the M.Sc. degree from the Universidad de Castilla-La Mancha, Ciudad Real, Spain, in 2012 and 2013, respectively. He is currently pursuing the Ph.D. degree at the Universidad de Castilla-La Mancha.

His research interests are in the fields of power systems planning, operations, and economics.

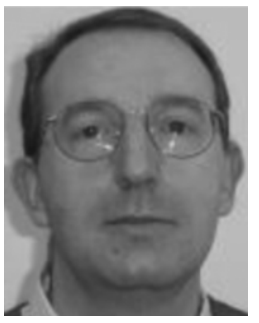

Javier Contreras (SM'05, F'15) received the B.S. degree in electrical engineering from the University of Zaragoza, Zaragoza, Spain, in 1989, the M.Sc. degree from the University of Southern California, Los Angeles, in 1992, and the Ph.D. degree from the University of California, Berkeley, in 1997.

He is currently Full Professor at the University of Castilla - La Mancha, Ciudad Real, Spain. His research interests include power systems planning, operations and economics, and electricity markets. 